\title{
Imaging mitotic processes in three dimensions with lattice light-sheet microscopy
}

\author{
Yuko Mimori-Kiyosue (D)
}

Received: 11 January 2021 /Revised: 17 February 2021 / Accepted: 19 February 2021 / Published online: 11 March 2021

(C) The Author(s) 2021

\begin{abstract}
There are few technologies that can capture mitotic processes occurring in three-dimensional space with the desired spatiotemporal resolution. Due to such technical limitations, our understanding of mitosis, which has been studied since the early 1880 s, is still incomplete with regard to mitotic processes and their regulatory mechanisms at a molecular level. A recently developed high-resolution type of light-sheet microscopy, lattice light-sheet microscopy (LLSM), has achieved unprecedented spatiotemporal resolution scans of intracellular spaces at the whole-cell level. This technology enables experiments that were not possible before (e.g., tracking of growth of every spindle microtubule end and discrimination of individual chromosomes in living cells), thus providing a new avenue for the analysis of mitotic processes. Herein, principles of LLSM technology are introduced, as well as experimental techniques that became possible with LLSM. In addition, issues remaining to be solved for use of this technology in mitosis research, big image data problems, are presented to help guide mitosis research into a new era.
\end{abstract}

Keywords Lattice light-sheet microscopy (LLSM) · live imaging $\cdot$ mitosis $\cdot$ chromosome $\cdot$ microtubules $\cdot$ EB1 organelles $\cdot$ cell shape

Responsible Editors: Kiichi Fukui and Toshiyuki Wako

Y. Mimori-Kiyosue $(\bowtie)$

Laboratory for Molecular and Cellular Dynamics, RIKEN Center for Biosystems Dynamics Research, 2-2-3

Minatojima-minamimachi, Chuo-ku, Kobe 650-0047, Japan

e-mail: yuko.kiyosue@riken.jp

\section{Introduction}

Capturing dynamic processes of mitosis at the wholecell level is necessary to understand how cells coordinate various events, such as attachment of spindle microtubules to kinetochores, interactions between astral microtubules and the cell cortex, and chromosome congression and separation. However, insufficient performance of conventional imaging techniques in terms of resolution and speed has crucially limited what can be observed (Liu et al. 2015).

In the seventeenth century, Hooke (Hooke 1665), van Leeuwenhoek (Leeuwenhoek 1682), and others discovered the "cell" using a newly developed device, the "microscope." In the early 1880 s, the pioneering studies of mitosis were started by German anatomist Walther Flemming (Flemming 1882; Mitchison and Salmon 2001; Paweletz 2001), who coined the term mitosis (thread in Greek) from the shape of mitotic chromosomes. More than half a century later, the first observation of a spindle apparatus within a living cell was made by W. J. Schmidt and Sinya Inoué, who used polarizing light microscopy (Inoué 1953; Schmidt 1939).

In this long history of cell biology of mitosis, however, whole-cell three-dimensional (3D) live imaging with sufficient spatiotemporal resolution to discriminate nanoscale-sized mitotic machineries and completely track their movements has never been achieved. The problem becomes particularly relevant when attempting to image subcellular dynamics using fluorescence microscopy (e.g. by introducing green fluorescent protein (GFP) technology (Tsien 1998; Zimmer 2002)), which 
enables systematic imaging of protein localization in living cells, as well as the structure and function of living tissues. Unfortunately, traditional imaging tools such as confocal microscopy, which uses an epiillumination configuration that exposes the entire sample thickness to illuminating radiation (Fig. 1a, left), induce out-of-focus background signals that reduce signal-to-noise ratio and increase phototoxicity (Icha et al. 2017; Laissue et al. 2017) and fluorescence photobleaching, and are often too slow to study minute subcellular processes in 3D detail across the entire cell volume. In addition, resolution in an epi-illumination configuration is substantially worse in the axial direction than the lateral plane (Gustafsson and Sedat 1999), which hampers precise measurement of 3D information.

Unlike popular epi-illumination, plane illumination microscopy limits excitation to the vicinity of the focal plane (Fig. 1a, middle and right), which provides effective optical sectioning and high-speed acquisition while minimizing out-of-focus background and photobleaching/phototoxicity (Huisken and Stainier 2009; Mertz 2011; Pampaloni et al. 2007; Planchon et al. 2011; Wan et al. 2019). Conventional light-sheet microscopes using Gaussian beams that are several microns thick were optimized for large specimens, such as small embryos and tissues (Fig. 1b, left). Recently, LLSM using an ultrathin $(\sim 0.4-1 \mu \mathrm{m})$ light sheet was developed (Fig. 1b, right), yielding superior signal-tobackground ratios, reduced photobleaching/phototoxicity, and ultrafast plane-wise imaging rates that enable whole-cell 3D scanning, often at subsecond intervals (Chen et al. 2014).

In this review, technical principles of LLSM and examples of its application are introduced to demonstrate how LLSM surpasses conventional microscopic technologies. In addition, critical challenges in image and data analysis that require solving to innovate future mitosis research using LLSM technology are discussed.

\section{Principles and advantages of LLSM}

LLSM is an advanced type of light-sheet microscopy (Chen et al. 2014; Wan et al. 2019). The advantage of light-sheet illumination is that it makes 3D live-imaging approaches to collect precise and reliable 3D information feasible. Unlike frequently used point-scanning techniques, such as confocal and two-photon microscopy, light-sheet microscopes illuminate specimens as a plane with a thin laser beam, while simultaneously imaging this plane with a camera in one shot (Fig. 1a). This approach achieves a combination of crucial properties for live imaging: high imaging speed and signal-to-noise ratio, and low energy load on the specimen, which minimize photobleaching and phototoxicity; collectively, these features allow for long-term imaging (Ahrens et al. 2013; Huisken and Stainier 2009; Keller et al. 2008; Krzic et al. 2012; Mertz 2011; Pampaloni et al. 2007; Planchon et al. 2011; Truong et al. 2011; Wan et al. 2019).

Remarkably, LLSM using an ultrathin light sheet (Fig. 1a, right and 1b, right) enables whole-cell 3D live imaging at unprecedented spatiotemporal resolution for extended periods of time. This is achieved by scans of cellular 3D volumes at subsecond intervals at a resolution of $\sim 230 \mathrm{~nm}$ laterally and $\sim 370 \mathrm{~nm}$ axially (Chen et al. 2014). In contrast to the conventional light sheet created by several micron-thick Gaussian beams (Fig. $1 \mathrm{~b}$, left), the lattice light sheet is generated by a massive parallel array of non-diffracting light beams (Bessel beams) that mutually interfere to create an ultrathin light sheet $\sim 0.4-1 \mu \mathrm{m}$-thick extending over cellular dimensions (Fig. 1b, right) (Chen et al. 2014). A Bessel beam is a special class of non-diffracting beam that allows a narrow beam width to propagate without spreading over a long distance due to the self-interference effect of the beam (Fig. 1b, middle) (Durnin 1987; Durnin et al. 1987; Planchon et al. 2011). Scanning with an ultrathin light sheet minimizes fluorescence photobleaching and phototoxicity, permitting acquisition of high-resolution images of whole cells for hundreds of volumes (Chen et al. 2014; Gao et al. 2012; Planchon et al. 2011). In the original LLSM model, the excitation beams are illuminated diagonally through a water-dipping objective lens (Fig. 1a, right) to place it in close proximity to specimens. However, a model with an inverted type configuration using specialized lenses has recently been commercialized.

Another important advantage of LLSM is that, unlike conventional super-resolution microscopy techniques such as stimulated emission depletion (STED) microscopy and photoactivated localization microscopy (PALM), it does not require the use of special fluorescent dyes or proteins. STED microscopy functions by depleting fluorescence surrounding the excitation focal spot while leaving a center spot active to emit fluorescence; thus, appropriate choices of dyes and lasers are critical to maximize performance (Dyba et al. 2003). For PALM, photo-switchable dyes or fluorescent proteins 
a

\section{Widefield epi-illumination}

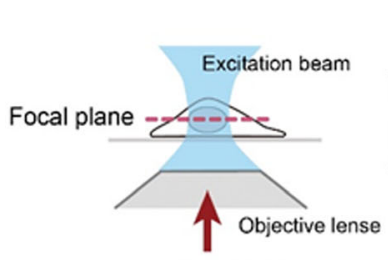

Observation axis

b

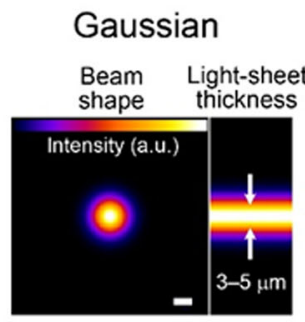

Gaussian light-sheet
plane-illumination

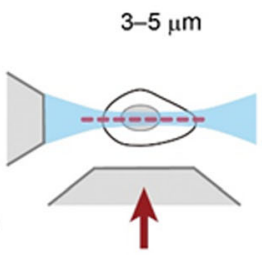

Single Bessel

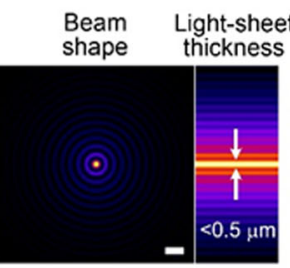

Bessel beam, lattice light-sheet plane-illumination

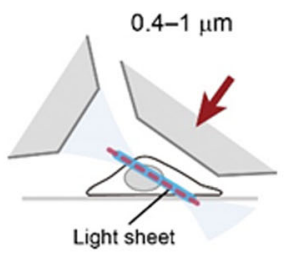

\section{Tiled acquisition and computational separation of cells}

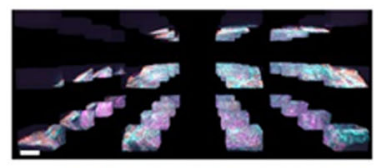

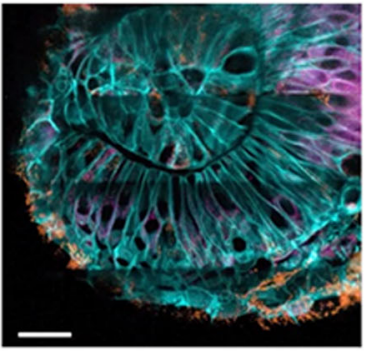

Fig. 1 Schematic comparisons of the optical sectioning power and beam shapes used in different illumination techniques, and methods for large volume imaging with LLSM. a Optical sectioning power of wide-field epi-illumination, Gaussian light-sheet, and Bessel beam/lattice light-sheet plane illumination techniques. Thickness of irradiation areas are shown in light blue. b Beam shapes of three different light-sheet techniques. Light-sheet thickness is shown on the right of each panel. Left: A Gaussian beam is used in traditional laser-scanning light-sheet microscopy (Keller et al. 2008). To create a virtual light-sheet, the beam is swept rapidly, providing time-averaged uniform illumination. Middle: Scanned Bessel beams in conjunction with structured illumination and/or two-photon excitation to create thinner light sheets $(<0.5$

are required to localize individual fluorophores by repeating the process of photoactivation, measurement, and bleaching of isolated single molecules over numerous cycles (Betzig et al. 2006). Therefore, dyeindependent LLSM has a wide range of applications

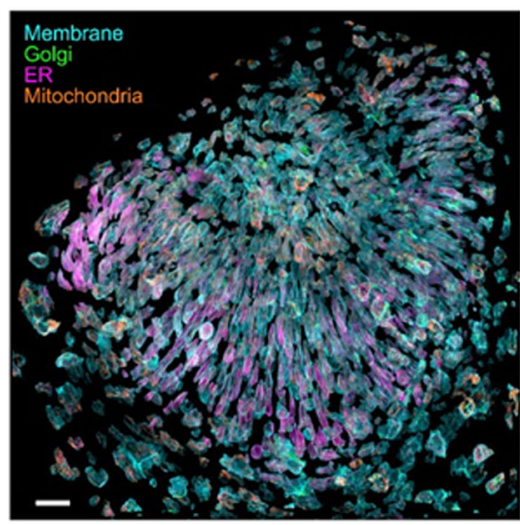

$\mu \mathrm{m})$ (Planchon et al. 2011). Right: Multiple Bessel beams arranged in a lattice pattern increases the sample scanning speed (Chen et al. 2014). Scale bars: $1 \mu \mathrm{m}$. Adapted from Ref. Chen et al. 2014. c Tiled acquisition and computational separation of each cell to image large volumes with LLSM. Upper left: Tiled array across the eye of a developing Zebrafish embryo (upper left). Scale bar: $30 \mu \mathrm{m}$. Lower left: Multiple subvolume tiles were stitched to generate the large volume image. Scale bar: $20 \mu \mathrm{m}$. Right: Computationally separated cells across the eye in a virtual space to visualize individual cells. Pseudocoloring of the image is indicated by the color of the characters. Scale bar, $30 \mu \mathrm{m}$. Adapted from Ref. Liu et al. 2018 under the Copyright (5004591427363)

including highly flexible multicolor imaging (Valm et al. 2017), fluorescence resonance energy transfer imaging (O'Shaughnessy et al. 2019), expansion microscopy (Gao et al. 2019), and use of existing various transgenic animal models. 


\section{Image collection by LLSM}

LLSM offers two imaging modes: super-resolution structured illumination microscopy (SIM) and highspeed dithered (Chen et al. 2014; Li et al. 2015). In SIM mode, more than $2003 \mathrm{D}$ volumes can be acquired at 4-s intervals at a resolution of $150 \times$ $280 \mathrm{~nm} x z$, and multiple images are collected at each $z$-plane. In dithered mode, the two-dimensional (2D) lattice pattern is oscillated using a galvanometer to provide time-averaged uniform illumination, and only one $2 \mathrm{D}$ image at each $z$-plane is acquired at a rate of up to 100-200 frames per second and resolution of $230 \mathrm{~nm}$ in $x$ and $\sim 370 \mathrm{~nm}$ in $z$. This is $\sim 1.3-1.5$ times poorer in each direction than SIM mode, but dithered lattices achieve about 7.5-times faster imaging at a comparable signal-to-noise ratio. Furthermore, use of dithered mode to collect single-plane images at each z-plane minimizes photobleaching and phototoxicity, enabling data collection for thousands of time points. Overall, the dithered lattice light sheet is advantageous for most biological phenomena, including mitosis, unless additional resolution is required.

A more recent LLSM model with adaptive optics (AO), which correct for sample-induced aberrations in multicellular specimens (Booth 2007; Ji et al. 2010; Royer et al. 2016; Wang et al. 2014), was used to visualize mitotic processes in large multicellular volumes, such as organoids and Zebrafish embryos (Liu et al. 2018). Spatiotemporal resolution and noninvasiveness of this system are comparable to the original LLSM model, but tiled acquisition collects a larger volume (e.g., $>200 \times 200 \times 100 \mu \mathrm{m}$ ), thus covering a wide range of developing tissues (Fig. 1c, left). To visualize individual cells within the crowded multicellular environment of the intact organism, the plasma membrane staining was used to computationally separate each cell in a virtual space and isolate any desired cell (Fig. 1c, right).

The ability of LLSM technology to generate large amounts of image data (easily reaching $100 \mathrm{~GB}$ to $1 \mathrm{~TB}$ ) for each time lapse along with thousands of numerical datasets results in far more information than can be analyzed by conventional approaches. This newly emerging "big image data" problem is a critical challenge that must immediately be resolved, as will be discussed in a later section.

\section{Imaging of mitotic processes with LLSM}

The remarkable improvement in axial resolution of LLSM provides, for the first time, a complete overview of whole mitotic cells as a time-lapse video with submicron 3D resolution (Fig. 2). Importantly, this information can be observed from any viewing angle with similar resolving power by simply rotating the image (Chen et al. 2014; Liu et al. 2018; Yamashita et al. 2015; Yamashita et al. 2020). Such 3D representations of data clearly demonstrate the advantages of LLSM over conventional laser confocal methods (Fig. 2). In this section, examples of mitotic processes visualized with LLSM over a wide range of scale are introducedfrom single cultured cells to cells constituting developing tissues.

Remodeling of organelles during mitosis

At the onset of mitosis, every membrane-bordered organelle, including mitochondria, Golgi apparatus, and endoplasmic reticulum (ER) including the nuclear envelope, undergo vast rearrangements to be accurately inherited and delivered to their proper locations in daughter cells (Warren and Wickner 1996). This process has fascinated cell biologists since the early 1900s, when specific cytochemical markers capable of labeling individual organelles were first developed for light microscopy (Wilson 1925). Although determining how theses organelles transform their architecture in detail remains of great interest, such studies are challenging because of their complex 3D morphology and the rapidity of changes in cell organization as cell division progresses.

Chen et al. labeled LLC-PK1 cells by stable transfection with mEmerald-ER and mApple-H2B (histone H2B), stained them with MitoTracker Deep Red dye, and recorded $3003 \mathrm{D}$ volumes at 3.8-s intervals using LLSM (Chen et al. 2014) (Fig. 3a). As previously observed, mitochondria divided during mitosis (Taguchi et al. 2007) and the ER reorganized into large, extended cisternae, such that little remained of the reticular network observed during interphase ( $\mathrm{Lu}$ et al. 2009). Additionally, LLSM revealed that mitochondrial fragments are corralled by the ER cisternae as they move within and between these pockets. However, exchange of fluorophore between mitochondria and the $\mathrm{ER}$, suggesting their mutual fusion, was not observed. 

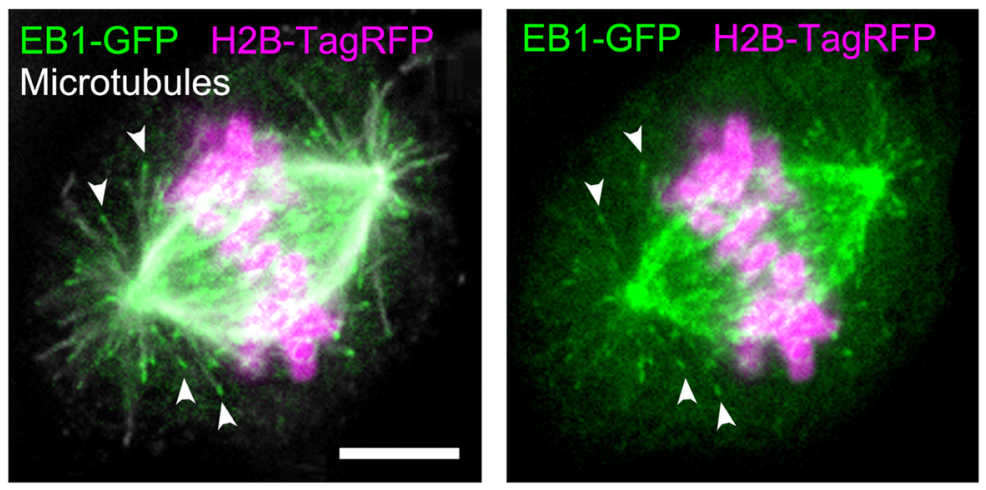

\section{LLSM image of a mitotic cell}

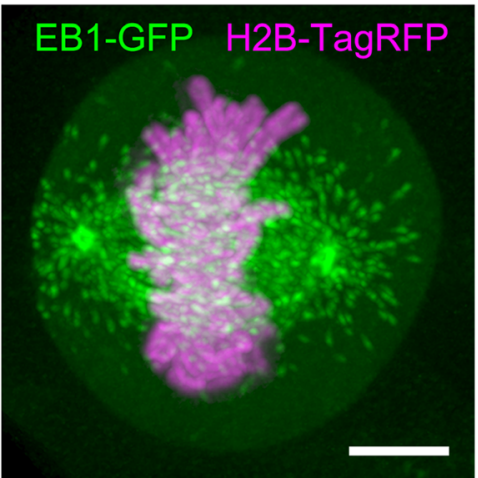

Fig. 2 Comparison of 3D stack images collected by conventional confocal microscopy and LLSM. a Distribution of EB1-GFP in a mitotic HeLa cell at metaphase. HeLa cells (clone A1) stably expressing EB1-GFP (green) and H2B-TagRFP (magenta) (Chen et al. 2014) were fixed and immunostained for microtubules (white) to identify the positions of microtubule ends. Z-stack images were collected under a conventional laser-scanning confocal microscope. Scale bar: $5 \mu \mathrm{m}$. Data is reused from Ref.

AO-LLSM was used to study various 3D subcellular processes throughout the cell cycle across populations of cells in vivo (Liu et al. 2018). To simultaneously examine the dynamics of multiple organelles throughout the cell cycle across a population of cells in Zebrafish embryos, brain progenitor cells with markers for transGolgi, ER, mitochondria, and plasma membrane were imaged (Fig. 3b). To visualize individual cells, the cells were computationally separated and desired cells were isolated. In interphase, multiple trans-Golgi segments were observed, which often appear as long filaments preferentially aligned along the axis of cell polarization (Fig. 3b, left) that fragmented during mitosis (Fig. 3b, right). The ER formed a reticular network in interphase and sheet-like cisternae during mitosis, as observed in

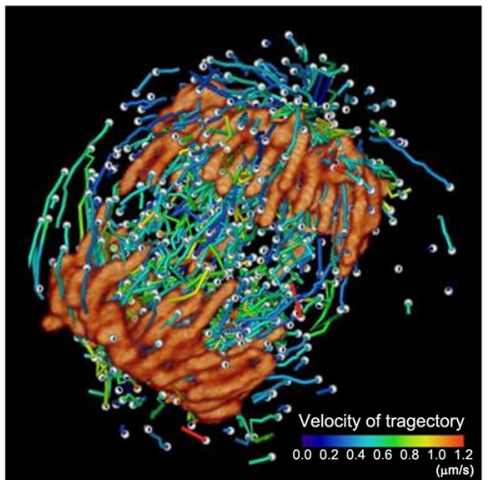

Yamashita et al. 2015. b Images collected by LLSM (left) were displayed in a 3D space using Imaris software. Note that individual EB1 comets can be discriminated even inside a thick spindle. Scale bars: $5 \mu \mathrm{m}$. Data is reused from Ref. Yamashita et al. 2015. On the right, trajectories of EB1-GFP movement are shown as balls and lines. The colored bar indicates the range of the mean travel speed of EB1-GFP comet trajectories $(0.0-1.2 \mu \mathrm{m} / \mathrm{s})$. Adapted from Ref. Chen et al. 2014

cultured cells. Mitochondria formed punctate structures near the surface and longer tubules in the subset of more deeply buried interphase cells (Fig. 3b, right). Mitochondria were preferentially located nearer the plasma membranes during mitosis, whereas other organelles were distributed uniformly.

3D tracking of spindle microtubule growth

Direct observation of individual filaments in the mitotic apparatus, which comprises hundreds of microtubule filaments (Inoué and Sato 1967; Inoué 1981; Salmon 1975), has never been achieved (Mitchison et al. 1986; Waterman-Storer et al. 1998) because of thickness of these structures and the high density of labeled protein. 
a Cultured LLC-PK1 cells

mApple-H2B MitoTracker mEmerald-ER

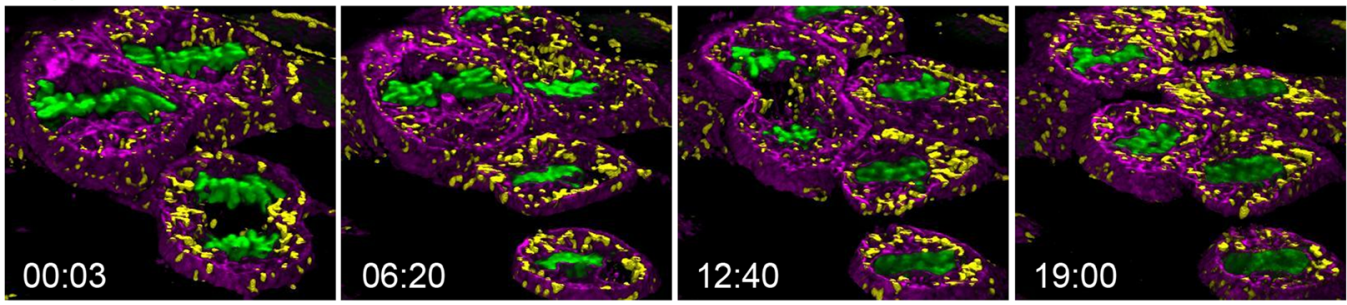

b Brain cells in a developing Zebrafish embryo

GalT-mNeonGreen (trans-Golgi) tagRFP-Sec61ß (ER) MitoTracker Citrine (plasma membrane)

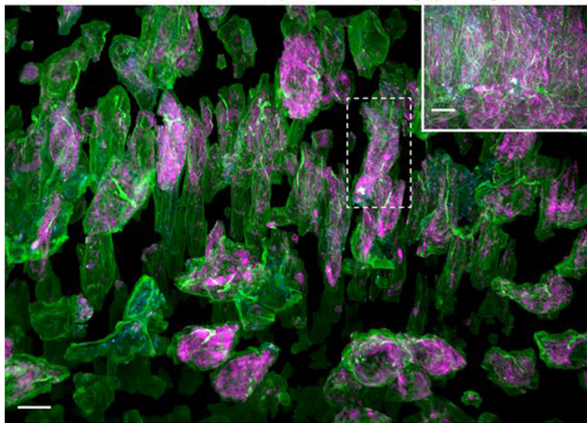

Fig. 3 Reorganization of organelles during mitosis visualized with LLSM. a Changing morphologies of histones (green), mitochondria (yellow), and ER (magenta) in dividing cultured LLCPK1 cells. Four time points in a slab extracted from a larger 3D time-series dataset imaged for 300 time points at 3.8-s intervals are shown. Adapted from Ref. Chen et al. 2014. Time scale: m:s. b Changing morphologies of trans-Golgi (green), ER (magenta),

Recently, we detected microtubule growth dynamics in $3 \mathrm{D}$ at the whole-cell level using LLSM (Chen et al. 2014; Yamashita et al. 2015) in conjunction with a GFP-conjugated form of the microtubule growth marker protein end-binding 1 (EB1-GFP), a microtubule plusend-tracking protein (Mimori-Kiyosue et al. 2000). EB1 binds selectively to the growing ends of microtubules in a comet-shape pattern with a short axis diameter of approximately $25 \mathrm{~nm}$ and anteroposteriorly elongated tail of up to $500 \mathrm{~nm}$ (Mimori-Kiyosue et al. 2000; Morrison et al. 1998). Notably, the utility of EB1-GFP as an analytical tool to study microtubule growth has been demonstrated in living cells and animals (Abe et al. 2011; Matov et al. 2010; Muroyama and Lechler 2017; Srayko et al. 2005). Time-lapse imaging and tracking of EB1-GFP comets yields microtubule growth trajectories, which permit analysis of microtubule growth rates, localization, and directionality (Applegate et al. 2011). However, until recently, image acquisition and analysis were predominantly limited to a $2 \mathrm{D}$ plane because of

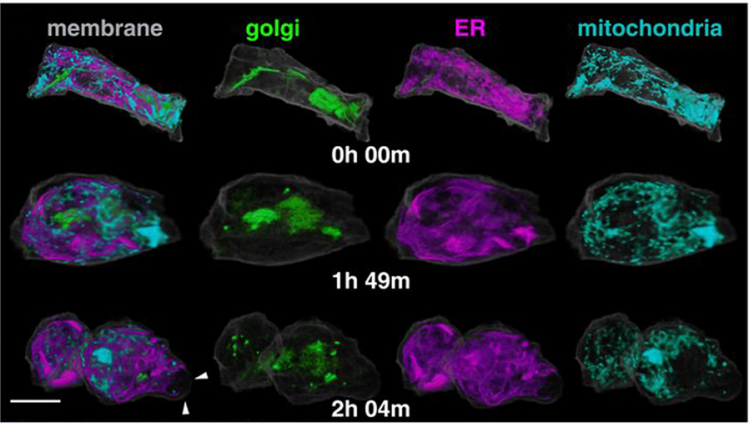

mitochondria (cyan), and plasma membrane (gray) in the brain of a developing Zebrafish embryo. Left: Computationally separated neural progenitor cells from a $70 \times 35 \times 35$ - $\mu \mathrm{m}$ region (inset) of a brain. Right: Changing morphologies of the organelles in the specific cell boxed in (a) at three time points through mitosis. Arrowheads indicate mitotic blebs. Scale bar, $10 \mu \mathrm{m}$. Adapted from Ref. Liu et al. 2018 under the Copyright (5004591427363)

insufficient spatiotemporal resolution of conventional fluorescence microscopes.

Using LLSM to generate a time-lapse sequence of mitotic HeLa cells expressing EB1-GFP and H2B-RFP at 0.755 -s intervals over a 56.625-s duration (75 timepoints) resulted in the detection of $>10,000$ EB1GFP comets and $>2000$ trajectories for each mitotic cell (Yamashita et al. 2015) (Fig. 4a-c). To analyze tracking results obtained as a large number of time-series $x-y-z$ coordinate datasets, we developed a data processing pipeline. Because the spindle apparatus often rotates and changes its orientation, changes in the angular orientation of the spindle were compensated using the centrosome positions as references to compare all datasets under a common reference frame. These data, "digital spindles" (Yamashita et al. 2020), can be used to generate an average image for each phase, classify trajectories by growth speed and position, and analyze growth speed and angles of individual microtubules in any area of interest (Fig. 4d). Such techniques are useful 


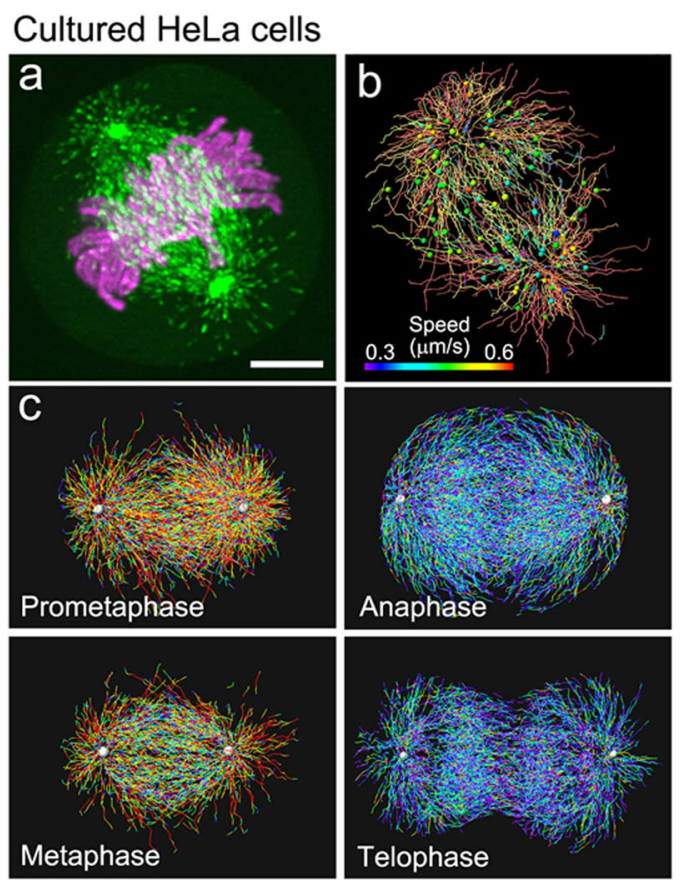

Fig. 4 Analysis of spindle microtubule growth as digital spindles. a A representative image of a HeLa cell (clone A1) expressing EB1-GFP (green) and H2B-TagRFP (magenta) from a time-series 3D dataset collected with LLSM. b An example of tracking of EB1-GFP comet movement. Trajectories are shown with colors corresponding to the average speed of each trajectory and dots at the end of the trajectory. The colored bar indicates the mean speed $(\mu \mathrm{m} / \mathrm{s})$. c Representative images of EB1-GFP trajectories at different mitotic phases. Trajectories were generated from 75 -frame

for precise phenotyping of cell division under different conditions, e.g., for comparison of normal and disease states (Kawasaki et al. 2020).

\section{Karyotyping in living cells}

Identification and tracking of individual chromosomes in living cells will reveal new insights into chromosome arrangements and how they change between healthy and diseased conditions. However, this has proven difficult for conventional epifluorescence methods including confocal microscopy due to inadequate axial resolution, the rounded shape and thickness of mitotic cells, and their sensitivity to light (Khodjakov and Rieder 2006). It is, however, a problem that can be solved by the high-resolution, low photobleaching/phototoxicity, and reduced out-of-focus background of ultrathin light-sheet illumination.

Gao et al. used super-resolution structured-plane illumination (SR-SIM) with a periodic Bessel beam (Bessel-plane SR-SIM) to image living U2OS cells

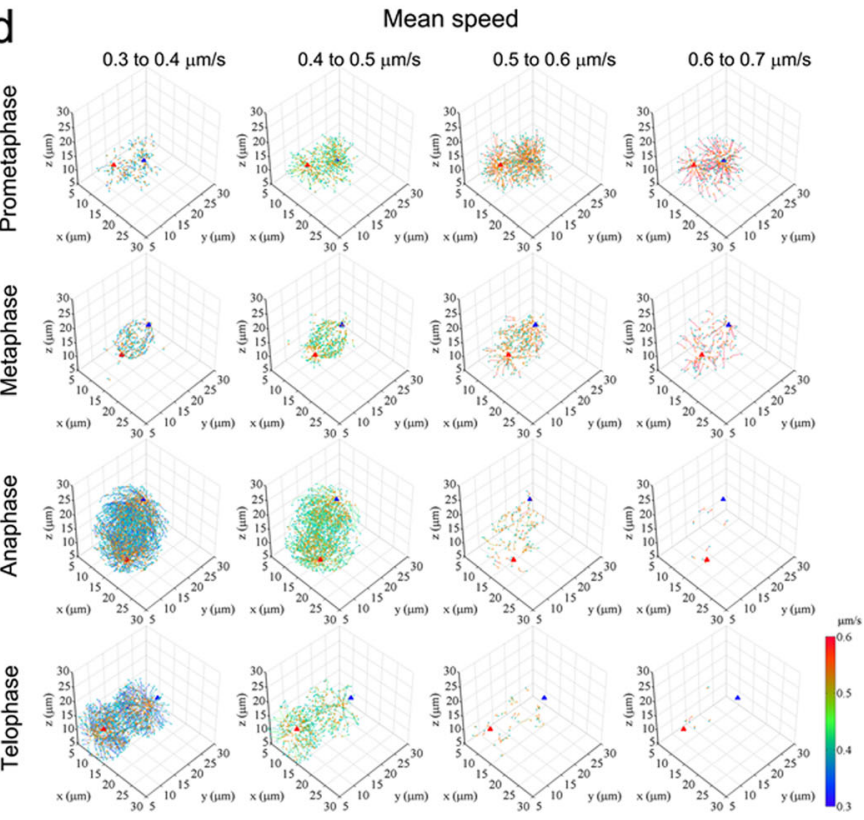

sequences corrected at 0.755 -s intervals. The color code is the same as shown in (b). d 3D distribution of EB1-GFP comet trajectories moving at different rates in each mitotic phase. Comet speed data were divided into 10 classes spanning the entire data range (0 to $1 \mu \mathrm{m} / \mathrm{s}$ ) using custom tools created in Matlab (Yamashita et al. 2015). Data included in the $0.3-0.7 \mu \mathrm{m} / \mathrm{s}$ range are shown in $3 \mathrm{D}$ coordinates. Blue and red triangles indicate centrosomes. Color bar indicates the mean speed $(\mu \mathrm{m} / \mathrm{s})$. Data are from Ref. Yamashita et al. 2015

expressing mEmerald-H2B and tdTomato-CENP-B, a kinetochore marker (Gao et al. 2012) (Fig. 5a, b). To improve wide-field SR-SIM and its application in thick living specimens, SR-SIM combines the tightly confined planar illumination of a periodically stepped Bessel beam with the principles of wide-field 3D super-resolution structured illumination microscopy (wide-field SR-SIM) (Gustafsson et al. 2008). In cells examined with SR-SIM, movement of individual chromosomes to the metaphase plate was observed in $3 \mathrm{D}$ (Fig. 5a). In this massively polyploid cell, all 77 chromosomes could be identified at any time point by manual segmentation of all chromosomes (Fig. 5b). In each case, kinetochore pairs correctly locating at their corresponding chromatids were used to identify specific chromosomes.

Gao et al. also observed mitosis in multicellular systems using Bessel-plane SR-SIM. In a living syncytial embryo of $D$. melanogaster, chromosomes of multiple cells within 10-20 microns of the surface could be 
Living cultured U2OS cells

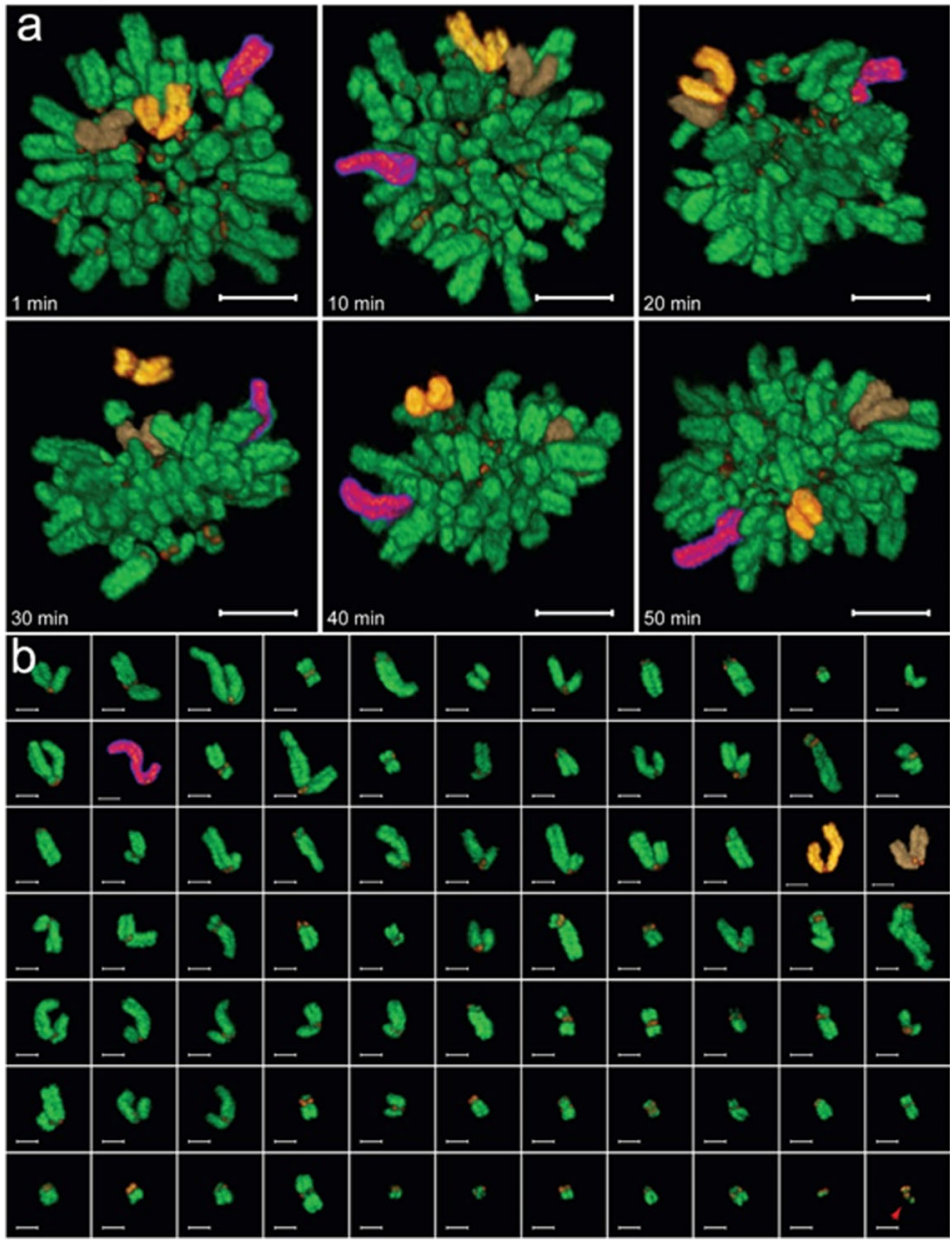

Living fly embryo
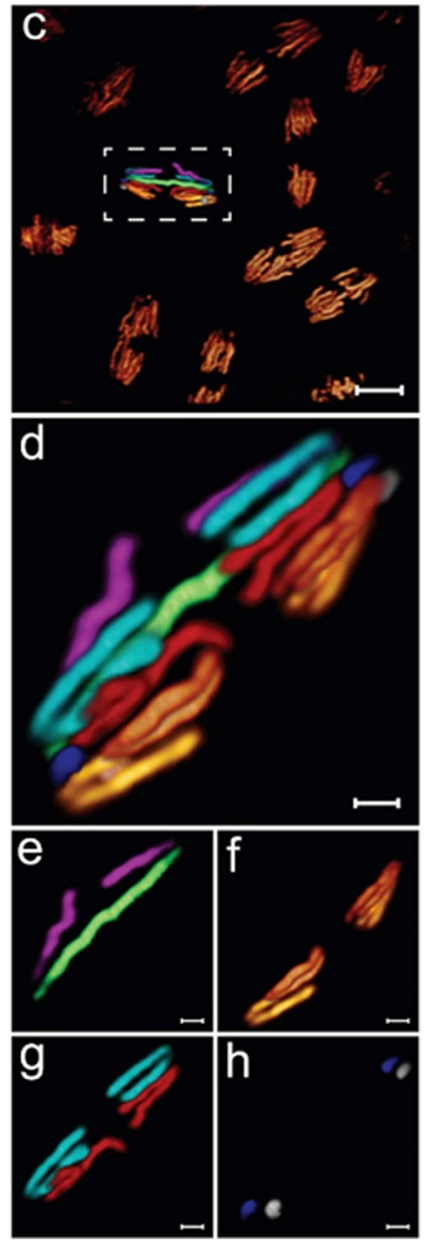

Fig. 5 Identification of individual chromosomes in cells. a, b Karyotyping of living cultured U2OS cells. Dual-color imaging of chromosomes (green) and kinetochores (red) at six time points from prometaphase to metaphase, selected from a series of 50 volumes acquired at 60-s intervals and collected using Besselplane SR-SIM (a). Three specific chromosomes (colored magenta, brown, and yellow) were identified by shape throughout the observation period. Karyotyping by manual segmentation of all chromosomes at the initial time point (b). Chromosomes were identified using the position of kinetochore pairs. The cell exhibits extreme polyploidy, with 76 diploids and one possible triploid (red arrow). Scale bars: $5 \mu \mathrm{m}$ in (a); $2 \mu \mathrm{m}$ in (b). c, d, e, f, $\mathbf{g}, \mathbf{h}$ Chromosomes in mitotic cells at the surface of a living syncytial embryo of $D$. melanogaster, visualized with Bessel beam plane SR-SIM (c). Enlarged view of the boxed chromosome in (c), in which individual chromosomes are differently colored (d). Sex chromosomes isolated from (d) identified the embryo as female (e), and autosomes 2, 3, and 4 could be identified ( $\mathrm{f}-\mathrm{h}$ ). Adapted from Ref. Gao et al. 2012 under the Copyright (5004601453014).

based on Bessel beam microscopy, a prototype of LLSM; thus, the improvements achieved by LLSM should yield similar or more accurate results, especially with LLSM SIM mode.

\section{Chromosomal passenger in early C. elegans embryos}

In early embryos of $C$. elegans, the cell cycle rapidly occurs by alternate repeating of $\mathrm{S}$ and $\mathrm{M}$ phases. An 
embryo expressing GFP-tagged Aurora B kinase homolog AIR-2, a chromosomal passenger complex protein (Carmena et al. 2012), was imaged with LLSM (Fig. 6). As previously reported, AIR-2::GFP mainly localized to metaphase chromosomes, followed by midbody microtubules in anaphase to telophase, and eventually in a persistent midbody remnant after cytokinesis (Schumacher et al. 1998). However, the thinness of the lattice light sheet allowed this process to be followed at a high signal-to-noise ratio with remarkable detail. AIR$2::$ GFP was first detected on chromosomes in prophase. By anaphase, it was most concentrated at spindle midzone microtubules, as previously observed, although some remained at the chromosomes and centrosomes, and faint signals were observed also along astral microtubules. High-spatiotemporal analysis of previously undetected distributions of mitosis regulators may provide new insight into how these molecules regulate cell division in developing embryos.

Morphology of dividing cells in different organs of developing Zebrafish embryos

Observation of different organs in Zebrafish embryos using AO-LLSM revealed phenotypic diversity across different cell types and developmental stages, as well as similarities and differences in mitosis progression, compared with cultured cells (Liu et al. 2018). The results revealed changes in the organization and position of organelles as cell division progresses, including the volume of cells and contained organelles. For example, formation of plasma membrane blebs observed transiently before cytokinesis in culture (Boss 1955; Laster and Mackenzie 1996) was commonly observed in various Zebrafish organs (Fig. 7). In contrast, although it has been shown that the shape and total surface of a cell change during mitosis in cultured cells (Aguet et al. 2016; Boucrot and Kirchhausen 2007), the total cellular volume remained constant throughout mitosis in the eye and ear observed with AO-LLSM (Fig. 7b).

\section{Big image data problem}

As mentioned above, LLSM technology provides far more information than can be analyzed by conventional approaches that depend on human eyes and manual editing. Efforts to tackle this newly emerged challenge in cell biology have just begun. Image analysis that requires manual editing of series of images containing high-resolution spatiotemporal information is very time consuming and data processing for a pipeline requires extensive calculations, making it difficult to routinely process a large number of datasets in typical biology

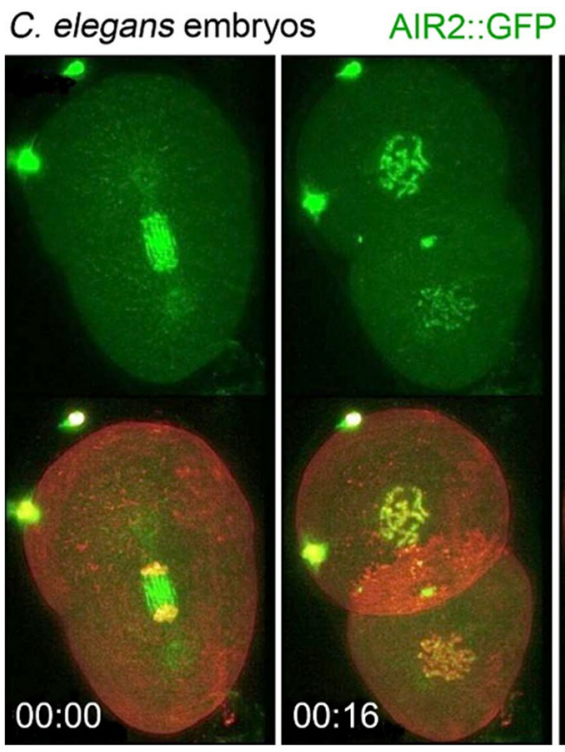

Fig. 6 Localization of the chromosomal passenger protein AIR2::GFP during cell divisions of the early $C$. elegans embryo. The thinness of the lattice light sheet allowed AIR-2::GFP distribution

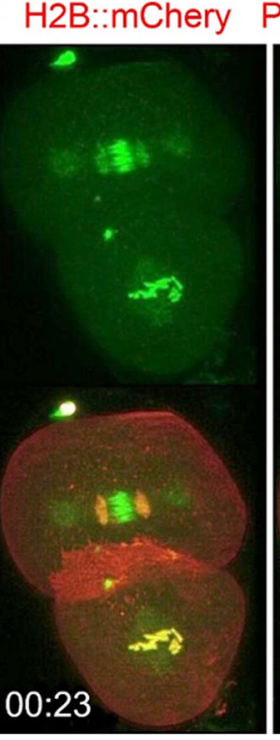

\section{Plasma Membrane::mCherry}

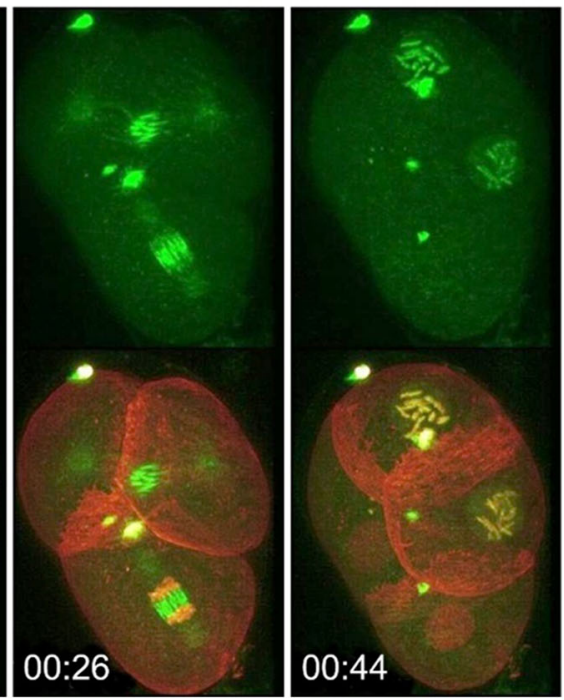

to be followed with remarkable detail. Time scale: h:m. Adapted from Ref. Chen et al. 2014 


\section{Eye of a developing Zebrafish embryo}
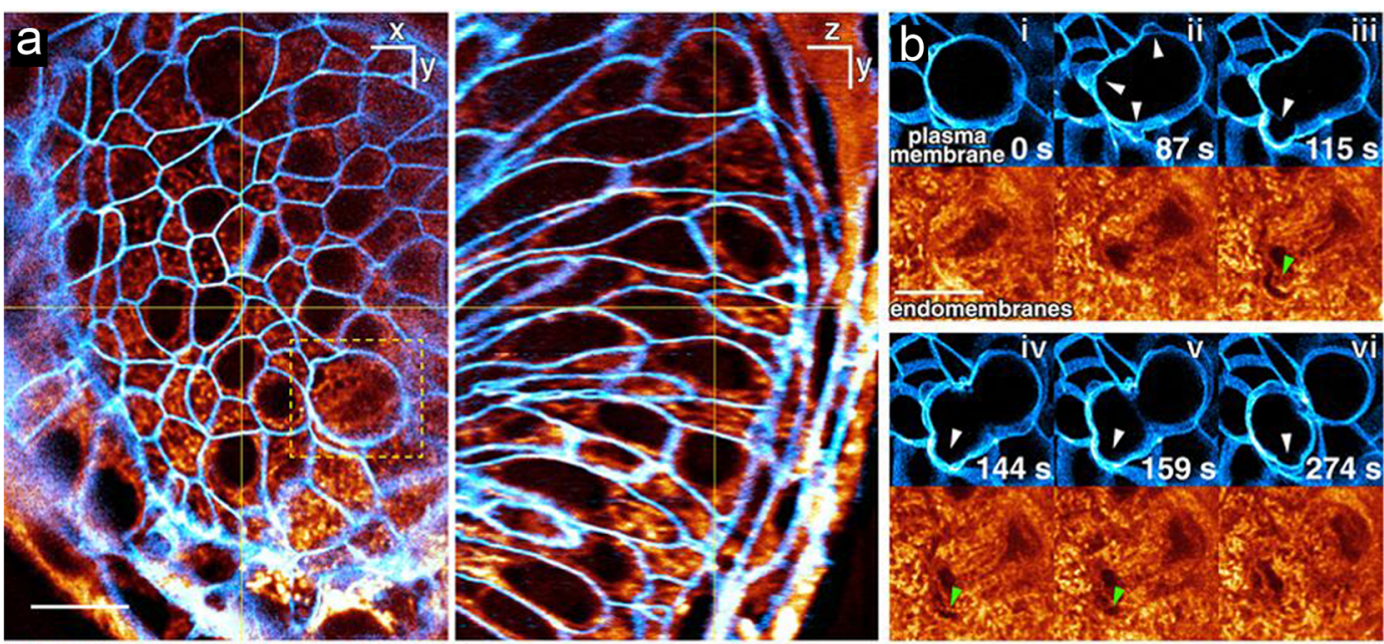

\section{Ear of a developing Zebrafish embryo}
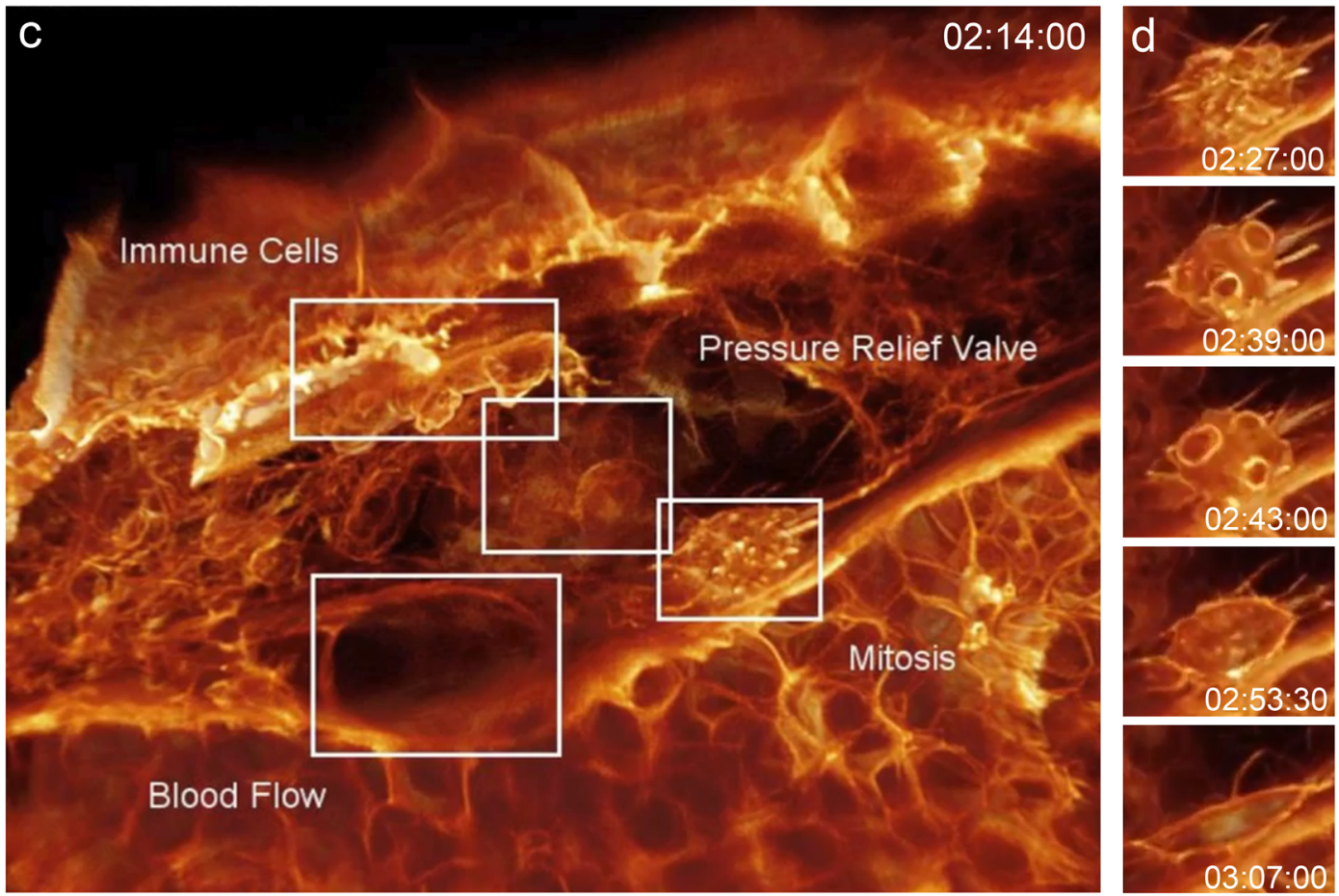

Fig. 7 Changing morphologies of the cell surface during mitosis in vivo visualized with LLSM. a, b Membranes in the developing Zebrafish eye from a time-series $3 \mathrm{D}$ video collected with AOLLSM at 43.8-s intervals for 200 time points. Plasma membrane visualized with membrane-citrins (blue) and endomembrane stained with Bodipy-tetramethylrhodamine (TMR) (orange), viewed as $1-\mu \mathrm{m}$-thick slab orthoslices (a). Six time points from the video showing plasma membrane blebs (white arrowheads) during mitosis and the exclusion of endomembranes in early blebs (green arrowheads). c, d A mitotic cell within the perilymphatic space next to the inner ear of a living transgenic Zebrafish embryo expressing membrane-Citrine. A $3 \mathrm{D}$ view of the tissue from a time-series 3D video collected for 438 time points at 13 -s intervals (a). Cells and structures observed are indicated. The morphology of mitotic cells at different five different time points (d), indicating production of transient blebs. Time scale: h:m:s. Adapted from Ref. Liu et al. 2018 under the Copyright (5004591427363) 
laboratories. Therefore, development of automated and user-friendly computational tools, and their adaptation for widespread use across biological research fields are essential for practical use of LLSM technology.

Recently, a method to generate a dynamic protein atlas of human cell division was reported (Cai et al. 2018). The authors automatically imaged mitotic cells in culture using a conventional confocal microscope, and then calibrated the signal by fluorescencecorrelation spectroscopy to convert protein fluorescence images to time-resolved distribution maps of protein concentrations. Using these data, they generated a canonical model of morphological changes of cells as mitosis progresses. Such an approach to time-series 3D volume data provides new opportunities for big image data mining.

In information science, one of the most successful advances in big data analysis in recent years has been deep-learning technologies (LeCun et al. 2015; Min et al. 2017; Shen et al. 2017). Ronneberger's group reported U-Net, a generic deep-learning technique for quantification tasks in biomedical image data, such as cell detection and shape measurements (Falk et al. 2019; Ronneberger et al. 2015). U-Net featuring pretrained models for single-cell segmentation can work on ImageJ software (Schneider et al. 2012), the most popular image analysis tool, thus enabling non-machine-learning experts to analyze data with only a few annotated samples as training data. However, the inspection and quantification of 3D images remains difficult, and tools for subcellular 3D morphometry have not been developed (Driscoll and Danuser 2015). Recently, a generic morphological-motif detector for 3D images capable of automatically identifying lamellipodia, filopodia, blebs, and other motifs using u-shape3D (a computer graphics and machine-learning pipeline) was reported (Driscoll et al. 2019). Extending this technology to analyze mitotic cells is a promising direction.

\section{Conclusions}

Recent innovations in high-resolution live-cell 3D microscopy using LLSM technology have begun to enable imaging of mitotic processes with unprecedented detail. This imaging technique provides a rich source of cellular information that far exceeds the information that cell biology has gained thus far. However, at present, no standardized methodologies for big image data analysis exist. Thus, incorporation of the latest information science technology to analysis of images and data extracted from the images is a critical and urgent challenge. Once a functional environment for big image data analysis has been established, this comprehensive measurement and analysis system will be a powerful tool for exploring novel molecular mechanisms regulating mitosis in normal and disease states, as well as high-throughput, highcontent screening for precise and accurate drug discovery.

Abbreviations $L L S M$, Lattice light-sheet microscopy; GFP, Green fluorescent protein; EB1, End-binding1; SR-SIM, Super-resolution structured illumination microscopy; $A O$,

Adaptive optics; ER, Endoplasmic reticulum; STED, Stimulated emission depletion; PALM, Photoactivated localization microscopy

Acknowledgements I thank Drs. N. Yamashita, M. Morita, and H. Yokota (Image Processing Research Team, RIKEN Center for Advanced Photonics, Saitama, Japan), and Drs. E. Betzig and W.R. Legant (HHMI, Janelia Research Campus, Ashburn, VA) for our earlier collaboration based on this review article, and for their generous support by providing technical information of LLSM, respectively.

Author contributions Y.M.-K. wrote the manuscript.

Funding Y.M.-K. was supported by the Japan Society for the Promotion of Science-NEXT program (LS128), the Takeda Science Foundation, the Uehara Memorial Foundation, a Grant-inAid for Challenging Exploratory Research (Japan Society for the Promotion of Science KAKENHI no. 20 K20379), and Japan Science and Technology Agency Core Research for Evolutional Science and Technology (no. JPMJCR1863), and an intramural grant from RIKEN.

\section{Declarations}

Conflict of interest The author declares that she have no conflicts of interest.

Open Access This article is licensed under a Creative Commons Attribution 4.0 International License, which permits use, sharing, adaptation, distribution and reproduction in any medium or format, as long as you give appropriate credit to the original author(s) and the source, provide a link to the Creative Commons licence, and indicate if changes were made. The images or other third party material in this article are included in the article's Creative Commons licence, unless indicated otherwise in a credit line to the material. If material is not included in the article's Creative Commons licence and your intended use is not permitted by statutory regulation or exceeds the permitted use, you will need to obtain permission directly from the copyright holder. To view a copy of this licence, visit http://creativecommons.org/licenses/by/4.0/. 


\section{References}

Abe T, Kiyonari H, Shioi G, Inoue K, Nakao K, Aizawa S, Fujimori T (2011) Establishment of conditional reporter mouse lines at ROSA26 locus for live cell imaging. Genesis 49:579-590. https://doi.org/10.1002/dvg.20753

Aguet F, Upadhyayula S, Gaudin R, Chou YY, Cocucci E, He K, Chen BC, Mosaliganti K, Pasham M, Skillern W, Legant WR, Liu TL, Findlay G, Marino E, Danuser G, Megason S, Betzig E, Kirchhausen T (2016) Membrane dynamics of dividing cells imaged by lattice light-sheet microscopy. Mol Biol Cell 27:3418-3435. https://doi.org/10.1091/mbc.E1603-0164

Ahrens MB, Orger MB, Robson DN, Li JM, Keller PJ (2013) Whole-brain functional imaging at cellular resolution using light-sheet microscopy. Nat Methods 10:413-420. https://doi.org/10.1038/nmeth.2434

Applegate KT, Besson S, Matov A, Bagonis MH, Jaqaman K, Danuser G (2011) plusTipTracker: Quantitative image analysis software for the measurement of microtubule dynamics. J Struct Biol 176:168-184. https://doi.org/10.1016/j. jsb.2011.07.009

Betzig E, Patterson GH, Sougrat R, Lindwasser OW, Olenych S, Bonifacino JS, Davidson MW, Lippincott-Schwartz J, Hess HF (2006) Imaging intracellular fluorescent proteins at nanometer resolution. Science 313:1642-1645. https://doi. org/10.1126/science. 1127344

Booth MJ (2007) Adaptive optics in microscopy. Philos Trans A Math Phys Eng Sci 365:2829-2843. https://doi.org/10.1098 /rsta.2007.0013

Boss J (1955) Mitosis in cultures of newt tissues. IV. The cell surface in late anaphase and the movements of ribonucleoprotein. Exp Cell Res 8:181-187. https://doi.org/10.1016 /0014-4827(55)90055-0

Boucrot E, Kirchhausen T (2007) Endosomal recycling controls plasma membrane area during mitosis. Proc Natl Acad Sci U S A 104:7939-7944.https://doi.org/10.1073 /pnas.0702511104

Cai Y, Hossain MJ, Hériché JK, Politi AZ, Walther N, Koch B, Wachsmuth M, Nijmeijer B, Kueblbeck M, Martinic-Kavur M, Ladurner R, Alexander S, Peters JM, Ellenberg J (2018) Experimental and computational framework for a dynamic protein atlas of human cell division. Nature 561:411-415. https://doi.org/10.1038/s41586-018-0518-z

Carmena M, Wheelock M, Funabiki H, Earnshaw WC (2012) The chromosomal passenger complex (CPC): from easy rider to the godfather of mitosis. Nat Rev Mol Cell Biol 13:789-803. https://doi.org/10.1038/nrm3474

Chen BC, Legant WR, Wang K, Shao L, Milkie DE, Davidson MW, Janetopoulos C, Wu XS, Hammer JA III, Liu Z, English BP, Mimori-Kiyosue Y, Romero DP, Ritter AT, Lippincott-Schwartz J, Fritz-Laylin L, Mullins RD, Mitchell DM, Bembenek JN, Reymann AC, Böhme R, Grill SW, Wang JT, Seydoux G, Tulu US, Kiehart DP, Betzig E (2014) Lattice light-sheet microscopy: imaging molecules to embryos at high spatiotemporal resolution. Science 346:1257998. https://doi.org/10.1126 /science. 1257998
Driscoll MK, Danuser G (2015) Quantifying modes of 3D cell migration. Trends Cell Biol 25:749-759. https://doi. org/10.1016/j.tcb.2015.09.010

Driscoll MK, Welf ES, Jamieson AR, Dean KM, Isogai T, Fiolka R, Danuser G (2019) Robust and automated detection of subcellular morphological motifs in 3D microscopy images. Nat Methods 16:1037-1044. https://doi.org/10.1038 /s41592-019-0539-z

Durnin J (1987) Exact solutions for nondiffracting beams. I. The scalar theory. J Opt Soc Am A Opt Image Sci Vis 4:651-654. https://doi.org/10.1364/JOSAA.4.000651

Durnin J, Miceli JJ Jr, Eberly JH (1987) Diffraction-free beams. Phys Rev Lett 58:1499-1501. https://doi.org/10.1103 /PhysRevLett.58.1499

Dyba M, Jakobs S, Hell SW (2003) Immunofluorescence stimulated emission depletion microscopy. Nat Biotechnol 21: 1303-1304. https://doi.org/10.1038/nbt897

Falk T, Mai D, Bensch R, Çiçek Ö, Abdulkadir A, Marrakchi Y, Böhm A, Deubner J, Jäckel Z, Seiwald K, Dovzhenko A, Tietz O, Dal Bosco C, Walsh S, Saltukoglu D, Tay TL, Prinz M, Palme K, Simons M, Diester I, Brox T, Ronneberger O (2019) U-Net: deep learning for cell counting, detection, and morphometry. Nat Methods 16:67-70. https://doi. org/10.1038/s41592-018-0261-2

Flemming W (1882) Zellsubstanz, kern und zelltheilung. FCW Vogel, Leipzig 1882:424

Gao L, Shao L, Higgins CD, Poulton JS, Peifer M, Davidson MW, Wu X, Goldstein B, Betzig E (2012) Noninvasive imaging beyond the diffraction limit of $3 \mathrm{D}$ dynamics in thickly fluorescent specimens. Cell 151:1370-1385. https://doi. org/10.1016/j.cell.2012.10.008

Gao R, Asano SM, Upadhyayula S, Pisarev I, Milkie DE, Liu TL, Singh V, Graves A, Huynh GH, Zhao Y, Bogovic J, Colonell J, Ott CM, Zugates C, Tappan S, Rodriguez A, Mosaliganti KR, Sheu SH, Pasolli HA, Pang S, Xu CS, Megason SG, Hess H, Lippincott-Schwartz J, Hantman A, Rubin GM, Kirchhausen T, Saalfeld S, Aso Y, Boyden ES, Betzig E (2019) Cortical column and whole-brain imaging with molecular contrast and nanoscale resolution. Science 363: eaau8302. https://doi.org/10.1126/science.aau8302

Gustafsson MG, Agard DA, Sedat JW (1999) I5M: 3D widefield light microscopy with better than $100 \mathrm{~nm}$ axial resolution. $\mathrm{J}$ Microsc 195:10-16. https://doi.org/10.1046/j.13652818.1999.00576.x

Gustafsson MG et al (2008) Three-dimensional resolution doubling in wide-field fluorescence microscopy by structured illumination. Biophys J 94:4957-4970. https://doi. org/10.1529/biophysj.107.120345

Hooke R (1665) Micrographia

Huisken J, Stainier DYR (2009) Selective plane illumination microscopy techniques in developmental biology. Development 136:1963-1975. https://doi.org/10.1242 /Dev.022426

Icha J, Weber M, Waters JC, Norden C (2017) Phototoxicity in live fluorescence microscopy, and how to avoid it. BioEssays 39. https://doi.org/10.1002/bies.201700003

Inoué S, Sato H (1967) Cell motility by labile association of molecules. The nature of mitotic spindle fibers and their role in chromosome movement. J Gen Physiol 50(Suppl):259292 
Inoué S (1953) Polarization optical studies of the mitotic spindle. 1. the demonstration of spindle fibers in living cells. Chromosoma 5:487-500. https://doi.org/10.1007 /Bf01271498

Inoué S (1981) Video image processing greatly enhances contrast, quality, and speed in polarization-based microscopy. J Cell Biol 89:346-356

Ji N, Milkie DE, Betzig E (2010) Adaptive optics via pupil segmentation for high-resolution imaging in biological tissues. Nat Methods 7:141-147. https://doi.org/10.1038 /nmeth.1411

Kawasaki Y et al (2020) APC mutant cells exploit compensatory chromosome alterations to restore tumour cell fitness. bioRxiv. https://doi.org/10.1101/2020.09.18.303016

Keller PJ, Schmidt AD, Wittbrodt J, Stelzer EH (2008) Reconstruction of zebrafish early embryonic development by scanned light sheet microscopy. Science 322:10651069. https://doi.org/10.1126/science.1162493

Khodjakov A, Rieder CL (2006) Imaging the division process in living tissue culture cells. Methods 38:2-16. https://doi. org/10.1016/j.ymeth.2005.07.007

Krzic U, Gunther S, Saunders TE, Streichan SJ, Hufnagel L (2012) Multiview light-sheet microscope for rapid in toto imaging. Nat Methods 9:730-733. https://doi.org/10.1038 /nmeth.2064

Laissue PP, Alghamdi RA, Tomancak P, Reynaud EG, Shroff H (2017) Assessing phototoxicity in live fluorescence imaging. Nat Methods 14:657-661. https://doi.org/10.1038 /nmeth.4344

Laster SM, Mackenzie JM Jr (1996) Bleb formation and F-actin distribution during mitosis and tumor necrosis factor-induced apoptosis. Microsc Res Tech 34:272-280. https://doi. org/10.1002/(SICI)1097-0029(19960615)34:3<272::AIDJEMT10>3.0.CO;2-J

LeCun Y, Bengio Y, Hinton G (2015) Deep learning. Nature 521: 436-444. https://doi.org/10.1038/nature14539

Leeuwenhoek V (1682) A. Letter no. 35

Li D et al (2015) Extended-resolution structured illumination imaging of endocytic and cytoskeletal dynamics. Science 349:aab3500. https://doi.org/10.1126/science.aab3500

Liu TL, Upadhyayula S, Milkie DE, Singh V, Wang K, Swinburne IA, Mosaliganti KR, Collins ZM, Hiscock TW, Shea J, Kohrman AQ, Medwig TN, Dambournet D, Forster R, Cunniff B, Ruan Y, Yashiro H, Scholpp S, Meyerowitz EM, Hockemeyer D, Drubin DG, Martin BL, Matus DQ, Koyama M, Megason SG, Kirchhausen T, Betzig E (2018) Observing the cell in its native state: imaging subcellular dynamics in multicellular organisms. Science 360:eaaq1392. https://doi.org/10.1126/science.aaq1392

Liu Z, Lavis LD, Betzig E (2015) Imaging live-cell dynamics and structure at the single-molecule level. Mol Cell 58:644-659. https://doi.org/10.1016/j.molcel.2015.02.033

Lu L, Ladinsky MS, Kirchhausen T (2009) Cisternal organization of the endoplasmic reticulum during mitosis. Mol Biol Cell 20:3471-3480. https://doi.org/10.1091/mbc.E09-04-0327

Matov A, Applegate K, Kumar P, Thoma C, Krek W, Danuser G, Wittmann T (2010) Analysis of microtubule dynamic instability using a plus-end growth marker. Nat Methods 7:761U134. https://doi.org/10.1038/Nmeth.1493
Mertz J (2011) Optical sectioning microscopy with planar or structured illumination. Nat Methods 8:811-819. https://doi. org/10.1038/nmeth.1709

Mimori-Kiyosue Y, Shiina N, Tsukita S (2000) The dynamic behavior of the APC-binding protein EB1 on the distal ends of microtubules. Curr Biol 10:865-868. https://doi. org/10.1016/S0960-9822(00)00600-X

Min S, Lee B, Yoon S (2017) Deep learning in bioinformatics. Brief Bioinform 18:851-869. https://doi.org/10.1093 /bib/bbw068

Mitchison T, Evans L, Schulze E, Kirschner M (1986) Sites of microtubule assembly and disassembly in the mitotic spindle. Cell 45:515-527

Mitchison TJ, Salmon ED (2001) Mitosis: a history of division. Nat Cell Biol 3:E17-E21. https://doi.org/10.1038/35050656

Morrison EE, Wardleworth BN, Askham JM, Markham AF, Meredith DM (1998) EB1, a protein which interacts with the APC tumour suppressor, is associated with the microtubule cytoskeleton throughout the cell cycle. Oncogene 17: 3471-3477. https://doi.org/10.1038/sj.onc. 1202247

Muroyama A, Lechler T (2017) A transgenic toolkit for visualizing and perturbing microtubules reveals unexpected functions in the epidermis. Elife 6. https://doi.org/10.7554 /eLife. 29834

O'Shaughnessy EC et al (2019) Software for lattice light-sheet imaging of FRET biosensors, illustrated with a new Rap1 biosensor. J Cell Biol 218:3153-3160. https://doi. org/10.1083/jcb.201903019

Pampaloni F, Reynaud EG, Stelzer EHK (2007) The third dimension bridges the gap between cell culture and live tissue. Nat Rev Mol Cell Biol 8:839-845. https://doi.org/10.1038 /Nrm2236

Paweletz N (2001) Walther Flemming: pioneer of mitosis research. Nat Rev Mol Cell Biol 2:72-75. https://doi. org/10.1038/35048077

Planchon TA, Gao L, Milkie DE, Davidson MW, Galbraith JA, Galbraith CG, Betzig E (2011) Rapid three-dimensional isotropic imaging of living cells using Bessel beam plane illumination. Nat Methods 8:417-423. https://doi.org/10.1038 /nmeth.1586

Ronneberger O, Fischer P, Brox T (2015) U-Net: convolutional networks for biomedical image segmentation. Med Image Comput Comput Assist Interv 9351:234-241. https://doi. org/10.1007/978-3-319-24574-4_28

Royer LA, Lemon WC, Chhetri RK, Wan Y, Coleman M, Myers EW, Keller PJ (2016) Adaptive light-sheet microscopy for long-term, high-resolution imaging in living organisms. Nat Biotechnol 34:1267-1278. https://doi.org/10.1038/nbt.3708

Salmon ED (1975) Spindle microtubules - thermodynamics of invivo assembly and role in chromosome movement. Ann N Y Acad Sci 253:383-406. https://doi.org/10.1111/j.17496632.1975.tb19216.x

Schmidt WJ (1939) Doppelbrechung der Kernspindel und Zugfasertheorie der Chromosomenbewegung Chromosoma 1:253-264

Schneider CA, Rasband WS, Eliceiri KW (2012) NIH Image to ImageJ: 25 years of image analysis. Nat Methods 9:671-675. https://doi.org/10.1038/nmeth.2089

Schumacher JM, Golden A, Donovan PJ (1998) AIR-2: an Aurora/Ipl1-related protein kinase associated with chromosomes and midbody microtubules is required for polar body 
extrusion and cytokinesis in Caenorhabditis elegans embryos. J Cell Biol 143:1635-1646. https://doi.org/10.1083 /jcb.143.6.1635

Shen D, Wu G, Suk HI (2017) Deep learning in medical image analysis. Annu Rev Biomed Eng 19:221-248. https://doi. org/10.1146/annurev-bioeng-071516-044442

Srayko M, Kaya A, Stamford J, Hyman AA (2005) Identification and characterization of factors required for microtubule growth and nucleation in the early $C$. elegans embryo. Dev Cell 9:223-236. https://doi.org/10.1016/j.devcel.2005. 07.003

Taguchi N, Ishihara N, Jofuku A, Oka T, Mihara K (2007) Mitotic phosphorylation of dynamin-related GTPase Drp1 participates in mitochondrial fission. J Biol Chem 282:1152111529. https://doi.org/10.1074/jbc.M607279200

Truong TV, Supatto W, Koos DS, Choi JM, Fraser SE (2011) Deep and fast live imaging with two-photon scanned lightsheet microscopy. Nat Methods 8:757-760. https://doi. org/10.1038/nmeth. 1652

Tsien RY (1998) The green fluorescent protein. Annu Rev Biochem 80(67):509-544. https://doi.org/10.1146/annurev. biochem.67.1.509

Valm AM, Cohen S, Legant WR, Melunis J, Hershberg U, Wait E, Cohen AR, Davidson MW, Betzig E, Lippincott-Schwartz J (2017) Applying systems-level spectral imaging and analysis to reveal the organelle interactome. Nature 546:162-167. https://doi.org/10.1038/nature22369

Wan Y, McDole K, Keller PJ (2019) Light-sheet microscopy and its potential for understanding developmental processes. Annu Rev Cell Dev Biol 35:655-681. https://doi. org/10.1146/annurev-cellbio-100818-125311
Wang K, Milkie DE, Saxena A, Engerer P, Misgeld T, Bronner ME, Mumm J, Betzig E (2014) Rapid adaptive optical recovery of optimal resolution over large volumes. Nat Methods 11:625-628. https://doi.org/10.1038/nmeth.2925

Warren G, Wickner W (1996) Organelle inheritance. Cell 84:395400. https://doi.org/10.1016/s0092-8674(00)81284-2

Waterman-Storer CM, Desai A, Bulinski JC, Salmon ED (1998) Fluorescent speckle microscopy, a method to visualize the dynamics of protein assemblies in living cells. Curr Biol 8: 1227-1230. https://doi.org/10.1016/S0960-9822(07)005155

Wilson EB (1925) the cell in development and heredity, 3rd edn. The Macmillan Co., New York

Yamashita N, Morita M, Legant WR, Chen BC, Betzig E, Yokota H, Mimori-Kiyosue Y (2015) Three-dimensional tracking of plus-tips by lattice light-sheet microscopy permits the quantification of microtubule growth trajectories within the mitotic apparatus. J Biomed Opt 20:101206. https://doi. org/10.1117/1.JBO.20.10.101206

Yamashita N, Morita M, Yokota H, Mimori-Kiyosue Y (2020) Digital spindle: a new way to explore mitotic functions by whole cell data collection and a computational approach. Cells 9. https://doi.org/10.3390/cells9051255

Zimmer M (2002) Green fluorescent protein (GFP): applications, structure, and related photophysical behavior. Chem Rev 102:759-781. https://doi.org/10.1021/cr010142r

Publisher's note Springer Nature remains neutral with regard to jurisdictional claims in published maps and institutional affiliations. 